\title{
Trascendencia de la formación docente de pandemia, aplicada para el regreso a la presencia.
} Reflexiones sobre la importancia de la formación docente durante la pandemia para el regreso a clases Transcendence of Pandemic Teacher Training Applied to the Return to Presence. Reflections on the Importance of Teacher Training during the Pandemic for Returning to School

Raúl Romero Lara Universidad Iberoamericana Ciudad de México, México raul.romero@ibero.mx

La formación y actualización de profesores ha sido un tema álgido, bien como parte de la práctica docente, un tema que pareciera por momentos urgente, bien como parte de una tendencia internacional o con base en decisiones políticas, e incluso como respuesta a emergencias climatológicas, geográficas o, como ha sucedido en el último año, producto de una situación sanitaria no prevista. Sin embargo, es una temática que, al menos en nuestro país, está presente desde la década de los ochenta.

Particularmente, el tema de la tecnología en la educación no sólo parece ser prioritario sino también urgente, necesario, pero a veces con sensación de agobio para los docentes; habrá que tomar en cuenta que éste no es un tema nuevo, la historia más reciente nos remonta a la televisión educativa de nuestro país, específicamente a la red de televisión educativa, Telesecundaria, que fue instaurada el 2 de enero de 1968 (Telesecundaria, s. f.). 
A más de 50 años de la implementación de la red EDUSAT, aún tenemos pendiente que nuestros profesores, nosotros como docentes $\mathrm{y}$ en todos los niveles educativos tengamos un manejo pertinente $\mathrm{y}$ eficiente de los recursos tecnológicos, para incorporarlos de manera crítica, pertinente y consciente en nuestra práctica, situación que ha cobrado enorme relevancia desde marzo de 2020, fecha en que ¡nos quedamos en casa!, para evitar riesgos, contagios y consecuencias trágicas, y momento en el que se hizo patente la diversidad, pero no por ello la eficiencia en la incorporación de las TIC en procesos educativos.

A más de un año y medio de la contingencia por Covid-19, maestros, estudiantes e instituciones tuvimos que migrar los recursos utilizados en procesos educativos presenciales, al uso de herramientas digitales para desarrollar nuestra práctica docente. Para algunos profesores esto fue una situación que apareció repentinamente, el trabajo en línea parecía ser un salto al vacío y aprender a usar un paracaídas mientras se desciende; para otros, retomar conocimientos previos o bien seguir con prácticas ya conocidas. Entre otras cosas, pretendieron evaluar los aprendizajes y procesos de los estudiantes de una manera tradicional, al solicitar una gran cantidad de trabajos escritos, muchas actividades, entre otros enviados a través de correo electrónico u otros medios que no necesariamente resultaron los más eficaces, lo que trajo consigo una saturación y agotamiento tanto de estudiantes como de docentes; aunado a esto, nos encontramos expuestos a muchas horas frente a la pantalla, sentados en una silla y con poca actividad física, pasando de la pantalla de la PC o laptop, a la de la TV abierta o de paga y rematando con la del celular o algún otro dispositivo móvil.

A docentes con cierta experiencia en el trabajo en línea, a distancia o con el uso de las Tecnologías de la Información y la Comunicación (TIC), quizá les fue más sencillo incorporar diversas herramientas digitales tanto para el trabajo y desarrollo de procesos educativos, como para la evaluación de aprendizajes conceptuales, procedimentales y actitudinales, siendo estos últimos, quizá, los menos atendidos, por lo pronto, al inicio de la contingencia. Algunos de estos recursos para evaluar han sido las rúbricas, los portafolios de evidencias y el seguimiento de participación a través algunas herramientas digitales. 
Lo cierto es que la incorporación de TIC no se ha dado de manera natural durante la contingencia, sino intempestiva e incluso invasiva dentro de lo urgente; para quienes contaban con poca preparación o experiencia, el correo electrónico ha sido la base para el envío y recepción de actividades de aprendizaje, en el mejor de los casos; también se han de recuperar las situaciones en las que los profesores implementaron pizarrones móviles en diversos vehículos para acercarse a estudiantes o bien buzones en las puertas de las escuelas, donde se intercambiaron actividades y entregas.

En este sentido, el acceso a la tecnología, lo mismo para docentes que para estudiantes, ha sido un punto importante a ser tomado en cuenta durante este periodo, así como la conectividad con la que cuentan las y los estudiantes en zonas rurales y urbanas. Si bien pudiera sobreentenderse que en las primeras la conectividad ha sido una limitante para el trabajo en línea, no es exclusivo de éstas; incluso en las grandes ciudades existen zonas donde la conexión deja mucho que desear.

En efecto, nos hemos encontrado con que el ancho de banda, la disponibilidad de datos y la arquitectura de los equipos tanto de estudiantes como de docentes, han jugado un papel importante, por lo que no es de extrańar que un recurso muy socorrido que han utilizado algunos profesores para el envío y recepción de tareas ha sido el WhatsApp, ya que las compañías de telefonía celular de nuestro país ofrecen este servicio, entre otros, de manera gratuita o a un bajo costo con un pago mínimo, a diferencia del uso de otros recursos, como el correo electrónico o el navegador de la Internet, que consumen muchos datos en la recepción y envío de archivos, o la descarga de diversos recursos, que implica un costo mayor.

Frente a esto, en marzo de 2020 nos encontramos con la necesidad de capacitación docente, específicamente en el uso de las TIC, situación un tanto emergente que nos tomó por sorpresa, si bien ya se venían promoviendo cursos o talleres de formación y actualización en esta área, la contingencia implicó que se atendiera a un mayor número de docentes en menor tiempo. Entre los principales retos que las instituciones enfrentaron para evaluar a los estudiantes de manera efectiva se encontró primero que los docentes migraran de una modalidad presencial a una mediada por 
tecnología, donde se promoviera el uso, la disponibilidad e incluso la creación de herramientas básicas y genéricas digitales.

Ante estos cambios de modalidad, tiempos y espacios, los docentes enfrentamos el reto de realizar evaluaciones a los estudiantes e incluso a la propia práctica docente. Ciertamente, entender que el medio es el mensaje, como dijo McLuhan (1969), ha sido un factor fundamental para tener una transición pertinente de lo presencial a lo digital, situación en la que el reto ha sido entender las características de estos recursos digitales para poder evaluar con base en el trabajo en línea ya sea en la modalidad a distancia, o híbrida, sincrónica o asincrónica, lo que ha implicado la formación de los docentes, por un lado, en el manejo de estos recursos, y por otro, en conocer las características de estas modalidades.

A su vez, el acceso a estos medios no ha sido homogéneo. Si bien la mayoría de la Instituciones de Educación Superior (IES) ya contaban con infraestructura, no podemos dejar de mirar que ésta se encuentra principalmente en las instalaciones de las propias instituciones y que los docentes y estudiantes ¡estamos en casa!, lo que implicó que mucho profesores tuvieron que adquirir, actualizar o mejorar tanto sus equipos de cómputo, como su servicio de conexión a la Internet, sin contar con el acondicionamiento mínimo del espacio donde laboran pero, además de esto, que los recursos han sido compartidos con el resto de los miembros de la familia.

Conforme se ha ido avanzando en el trabajo durante la pandemia, otro reto ha sido la organización e incluso la creación de recursos didácticos, como resultado de que los docentes ya se percataron de que, no por más trabajo o más tareas que dejen -más tiempo para calificar y menos descanso-, los estudiantes aprenderán mejor, situación que fue muy común al inicio de la pandemia, cuando docentes y estudiantes expuestos a largas jornadas frente a la pantalla sufrieron las consecuencias del agotamiento e incluso del desánimo.

Fue necesario desarrollar ambientes seguros y de confianza, en los que el acceso a nuestra intimidad y la del otro es una realidad pues, aunque ahora estamos en casa, la otredad se ha convertido en un elemento vital y el encuentro con el otro, en una experiencia fundamental en la construcción del conocimiento (Salas, 2012) con base en un enfoque humanista, otredad en la que el estudiante y el 
profesor descubren la existencia de otros con características propias, lo que permite reconocer y respetar al otro a través de interacciones que ahora, en la distancia, están mediadas por una pantalla.

A lo anterior hay que añadir la interculturalidad (Dietz, 2017), pues no solamente se reconoce al otro, sino que además se aprende de él, con él y junto a él en un proceso mutuo de construcción del conocimiento, en el que la colaboración es un hecho didáctico que incorpora la diversidad y la pluralidad de grupos y sectores a la comunidad estudiantil, que converge en un espacio común: la clase de aprendizaje. Ambos elementos, tanto la otredad como la interculturalidad deben ser, de ahora en adelante, fundamentales en nuestra práctica docente, por lo que al regreso a los espacios físicos deberemos continuar cuidando y revitalizando estos ámbitos seguros y de confianza, que contribuyan a procesos de enseńanza y de aprendizaje apoyados de recursos digitales o no digitales, y que promuevan el trabajo colaborativo y el aprendizaje significativo.

Es destacable que enfrentarse a la multipresencia, a las multipantallas ha sido todo un reto porque, si bien algunos jóvenes se consideran multitareas (Santori, 1997), la verdad es que se ha demostrado, durante este tiempo de pandemia, que en el desarrollo de las clases no pueden mantener la atención más que por periodos cortos cuando se tienen varias actividades abiertas o en elaboración simultánea, lo que implica la incorporación de diversos recursos y actividades variadas en tiempos cortos durante el desarrollo de las sesiones sincrónicas; por consiguiente, podemos afirmar que sí es necesario que estudiantes y docentes concentren su atención en la actividad que requiere su principal atención.

La contingencia por Covid-19 nos ha traído, indudablemente, aprendizaje y experiencias sobre diversas situaciones, tanto personales como profesionales. En lo que respecta a la evaluación de los aprendizajes, las formas y procesos han sido tan diversas como situaciones y contextos podemos imaginar; tan variadas como prácticas docentes podemos encontrar. Durante más de un año he aprendido sobre la incorporación que he realizado de diversos recursos y aplicaciones tanto en línea como descargables, para el trabajo sincrónico y asincrónico, lo que ha posibilitado que mi práctica docente esté en un proceso de mejora continua, avanzando hacia adelante, pero 
mirando por el retrovisor, como diría McLuhan (1969), me percato de que sí ha evolucionado.

Indudablemente, la gran mayoría de la IES han realizado un gran esfuerzo en la implementación de diversas estrategias, formas, medios y recursos para capacitar a sus docentes, no sólo en el uso de TIC como parte de su práctica cotidiana, sino en diversas herramientas para la evaluación de los aprendizajes de las y los estudiantes. A más de un año de contingencia, se reconoce que un gran aprendizaje fue valorar la formación y actualización continua de los maestros, con acciones que las instituciones deberán mantener, de ahora en adelante, como una estrategia de mejora permanente de la práctica de sus docentes y cada institución lo hace con base en su carisma, en el que comparte sus elementos fundacionales a sus docentes, quienes impregnan esta esencia en su identidad como profesores, y es por ello que la formación docente debe considerar y revisar su coherencia y correspondencia con su contexto inmediato.

En lo particular, este tiempo desarrollando mi práctica docente desde casa me ha permitido darme cuenta de que mi experiencia docente no sólo ha acumulado años, sino también gratas experiencias que me han permitido incorporar diversas herramientas y aplicaciones para desarrollar procesos de enseñanza y de aprendizaje dinámicos e interactivos, en los que la participación y colaboración de los estudiantes ha sido fundamental. En colectivo, también hemos identificado que la promoción de espacios flexibles para intercambiar experiencias entre docentes suele ser significativa y altamente formativa, a veces, más que los ámbitos temáticos, formales y rígidos, aquéllos donde permea la identidad institucional en los docentes (Romero y Guillen, 2019), con lo que se puede aspirar a una formación trascendental y con sentido para los maestros, correspondiente con su contexto, y características tanto de su infraestructura como la de las y los estudiantes.

Considero que, al regreso a las aulas de manera presencial o híbrida, se debe recuperar la experiencia de la capacitación y la actualización impartida durante este tiempo, para que sea una actividad continua; será vital incluir la importancia de formar para una planeación y dosificación de recursos y contenidos, tomando en cuenta las necesidades y condiciones del contexto local, nacional e interna- 
cional, así como de la infraestructura de los estudiantes. Esto permitirá una selección de herramientas, espacios o recursos pertinente y eficiente, lo que se podrá ver reflejado en la selección de estrategias de evaluación acordes con los contenidos, las estrategias y las metas, y permitirá valorar de forma pertinente y coherente los aprendizajes. Porque, aun entre estudiantes de la misma IES, de la misma zona geográfica, existen diferencias entre sus equipos de cómputo, dispositivos móviles e incluso respecto a su proveedor de servicio de conexión a la Internet. Retomar la experiencia que todos los docentes hemos tenido durante este tiempo a través de espacios flexibles, podría ser una estrategia mediante la que se involucre a todos los docentes en su propia formación y en la de sus compañeros.

Un gran reto a nivel nacional será revisar la infraestructura para la conectividad a la Internet, dado que $60.6 \%$ de los hogares la tiene, frente a $91.6 \%$ que tienen televisor (INEGI, 2020), lo que implica una desproporción de acceso a información y contenidos diversos. Cabe preguntarse ¿qué pasa con el 39.4\% de los hogares que aún no cuentan con conectividad?, ¿qué hicieron las niñas, los niños y jóvenes de estos hogares para continuar su proceso formativo durante la contingencia? Seguramente, al responder estas preguntas podremos encontrar historias de éxito de buenas prácticas docentes que acercaron a los estudiantes los contenidos educativos mediante estrategias que fueron desde pizarrones móviles, hasta buzones en las puertas de las escuelas e incluso visitas personalizadas.

Como se mencionó, se han identificado prácticas docentes que mediante el uso del Whats $A p p$ han tenido resultados interesantes en el desarrollo de las metas de aprendizaje, además de la cuestión de planes tarifarios o de renta, esto encuentra su razón de ser en que sólo $51.3 \%$ de usuarios de computadora usan esta herramienta como apoyo a sus actividades escolares (INEGI, 2020). Esto deja en claro que una proporción importante de estudiantes en los diversos niveles educativos ha accedido, por lo menos durante el último año, a los contenidos escolares a través de diversos dispositivos móviles como tabletas, teléfonos celulares, entre otros. Por eso será importante considerar que el diseño de recursos didácticos digitales multimedia, ya sea para su descarga o para el trabajo en línea, deberán ser accesibles y visibilizados a través de dispositivos con poca memoria 
RAM, procesadores de baja velocidad y pantallas que no necesariamente sean de última generación pues, por ejemplo, un video en calidad $4 \mathrm{~K}$ corre el riesgo de no poder ser visto por estudiantes cuyos dispositivos móviles no cuenten con la arquitectura para dicha descarga, lo que limitará el acceso a contenido y procesos formativos.

Por lo tanto, es importante considerar que acceder a plataformas robustas y llenas de animaciones, videos, y muchos archivos podría resultar ineficiente y poco pertinente para estudiantes que accedan a estos contenidos a través de dispositivos móviles, lo que se podría dificultar aún más si la conexión a la Internet es a través de un proveedor que distribuye un ancho de banda que no permita visualizar o descargar estos recursos. Esto evoca, para quienes hemos vivido la incorporación y desarrollo de las TIC, aquel recuerdo de querer descargar una imagen que tardaría varios minutos, y el riesgo de que alguien descolgara el teléfono e interrumpiera este proceso. Por lo anterior, es importante revisar, más no repetir, la evolución de las TIC en educación, en particular en nuestro país pues, como bien se dice, estudiar la historia nos permite no repetir los mismos errores y sí aprender de ellos.

Una vez que regresemos a las Universidades y las escuelas, valdrá la pena generar espacios de intercambio para recuperar las buenas prácticas que los docentes de todos los niveles hemos documentado y difundido a través de diversos medios, de las cuales podemos encontrar ejemplos de éxito en diversas redes sociales, como YouTube, con gran número de seguidores, sin olvidar las plataformas institucionales, donde también se pueden encontrar excelentes muestras tanto de recursos didácticos, como de estrategias de evaluación y de buenas prácticas docentes.

Esta experiencia pandémica nos lleva a tener presente que académicos, docentes y gestores nunca dejamos de aprender; ahora nos tocó en tópicos relacionados con las TIC, pero no debemos olvidar los fundamentos pedagógicos que nos permitan desarrollar procesos de enseñanza y de aprendizaje con mayor profundidad, en los que se promueva el pensamiento crítico y los retos intelectuales para estudiantes y para nosotros mismos, por lo que podría ser un buen momento para voltear a ver las principales instituciones de formación de docentes, las Escuelas Normales, de las de jardín de 
niños hasta las superiores, incluyendo las universidades que también imparten licenciaturas relacionadas con la formación docente, en carreras como pedagogía, por ejemplo, para pensar en una reforma o al menos en una actualización de contenidos que incluya de manera transversal la selección e implementación de TIC en procesos educativos y, por supuesto, en el diseño y elaboración de materiales propios, cuidando siempre el fomento de ambientes colaborativos y de aprendizaje contextualizados.

Tenemos frente a nosotros el reto de retomar la historia, y no como un acto de melancolía o nostalgia en el que recordemos que "antes se hacían mejor las cosas", sino como un referente que nos permita avanzar sin olvidar y teniendo presente cómo hemos evolucionado hasta la sociedad contemporánea, retomando cómo es que se han superado diversas vicisitudes, en particular en materia de emergencias en nuestro país, si bien la pandemia es una situación mundial, en nuestro contexto hemos salido avante en la historia cercana de un fuerte sismo, en 2017, cuando la tecnología ayudó a interactuar para poder acercar víveres, materiales de construcción y de curación a las zonas donde se requería, además de conectar familias que, por los daños en calles y de tránsito, no podían acercarse en lo inmediato, por lo que no podemos negar que las TIC son un recurso que requiere un uso crítico, creando contenido fundamentado en argumentos.

Por lo anterior, puedo afirmar que la formación y actualización que los docentes hemos tenido durante el tiempo de contingencia, ya sea por iniciativa propia o de manera institucional, es una herramienta que debe ser recuperada en este regreso a las aulas, quizá en espacios flexibles de reflexión y de compartir, o bien de manera estructurada, promoviendo relaciones y construcción de experiencias de manera horizontal.

No olvidemos que "para ver el futuro hay que ver el presente, apoyado en el pasado, esto es, ver los movimientos interiores, de las entrañas mismas. Por eso a la antropología filosófica le conviene un método hermenéutico, un camino interpretativo, para comprender lo íntimo del hombre, lo más entrañable que tiene" (Beuchot, 2004, p. 15). Tener presente que el centro de las IES, como instituciones sociales, es la persona, la formación de profesionistas capaces de inci- 
dir en la sociedad para la mejora continua de las condiciones de vida de los grupos en condiciones de vulnerabilidad y del bien común.

\section{REFERENCIAS}

Beuchot, M. (2014). Charles Sanders Peirce: semiótica, iconicidad y analogía. México: Editorial Herder.

Dietz, G. (2017). Interculturalidad: una aproximación antropológica. Perfiles educativos, XXXIX(156), 191-207.

INEGI (2020). Tecnologias de la Información y la Comunicación en Hogares. México: Gobierno de México. https://www.inegi.org.mx/temas/ ticshogares/

McLuhan, M. (1969). El medio es el mensaje. Argentina, Buenos Aires: Paidós.

Romero, R., y Guillen, J. (2019). Identidad docente, elemento de mejores prácticas docentes universitarias. XV Congreso Nacional de Investigación Educativa, Acapulco, México.

Salas, R. (2012). Intersubjetividad, otredad y reconocimiento. Diálogo fenomenológico para pensar la cuestión del otro en la filosofía intercultural. Cuadernos de la facultad de humanidades y ciencias sociales. Universidad nacional Jujuy, (42), 83-104. http://www.redalyc.org/articulo.oa?id=18531549006

Santori, G. (1997). El Homo videns, la sociedad teledirigida. Buenos Aires: Taurus, Alfaguara.

Telesecundaria (S/F). Historia. https://telesecundaria.gob.mx/historia. html 